\title{
A new acoustic assumption for orthorhombic media
}

\author{
Mohammad Mahdi Abedi ${ }^{*}$, Alexey Stovas ${ }^{2}$ \\ ${ }^{1}$ Basque Center for Applied Mathematics, Bilbao, Spain. E-mail: mabedi@bcamath.org \\ ${ }^{2}$ Norwegian University of Science and Technology, Department of Geoscience and Petroleum \\ Engineering, Trondheim, Norway.E-mail: alexey.stovas@ntnu.no \\ *Corresponding author
}

\section{SUMMARY}

In exploration seismology, the acquisition, processing and inversion of $\mathrm{P}$-wave data is a routine. However, in orthorhombic anisotropic media, the governing equations that describe the $\mathrm{P}$-wave propagation are coupled with two $\mathrm{S}$ waves that are considered as redundant noise. The main approach to free the $\mathrm{P}$-wave signal from the $\mathrm{S}$-wave noise is the acoustic assumption on the wave propagation. The conventional acoustic assumption for orthorhombic media zeros out the S-wave velocities along three orthogonal axes, but leaves significant S-wave artefacts in all other directions. The new acoustic assumption that we propose mitigates the S-wave artefacts by zeroing out their velocities along the three orthogonal symmetry planes of orthorhombic media. Similar to the conventional approach, our method reduces the number of required model parameters from nine to six. As numerical experiments on multiple orthorhombic models show, the accuracy of the new acoustic assumption also compares well to the conventional approach. On the other hand, while the conventional acoustic assumption simplifies the governing equations, the new acoustic assumption further complicates theman issue that emphasizes the necessity of simple approximate equations. Accordingly, we also propose simpler rational approximate phase-velocity and eikonal equations for the new acoustic orthorhombic media. We show a simple ray-tracing example and find out that the proposed approximate equations are still highly accurate.

Key words: Acoustic properties, Seismic anisotropy, Wave propagation, Numerical approximations and analysis

\section{INTRODUCTION}

In seismic data processing, modelling, and inversion, different assumptions are made about the wave propagation environments. Isotropy or a specific degree of symmetrical anisotropy are among these assumptions that are made based on the geological complexities, available model parameters, and computational complexities or costs.

In 3D models, orthorhombic (ORT) anisotropy has become a common model for the description of azimuthal and polar variations of seismic wave propagation. Different geological settings can result in an ORT anisotropy, among which, sets of vertical parallel fractures passing a finely layered sequence (Schoenberg \& Helbig 1997; Bakulin et al. 2000), or multiple

This paper is published by Geophysical Journal International: https://doi.org/10.1093/gji/ggaa367 
sets of parallel and orthogonal fractures (Bakulin et al. 2000) are two common patterns in exploration seismology.

While P-wave data processing and modelling is the most common case in exploration seismology, the equations that describe P-wave in ORT media are coupled with two S-waves. Requiring pure P-wave data in modelling and any other applications, these S-waves are considered as redundant noise. To free the P-wave data from the S-waves, an acoustic assumption is made on the wave propagation. For ORT media, such an acoustic assumption is proposed by Alkhalifah (2003). The Alkhalifah (2003) acoustic ORT assumption follows directly the Alkhalifah (1998) acoustic transversely isotropic (TI) assumption, which is built based on the weak sensitivity of P-wave kinematics on a parameter that describes S-wave velocity in the vertical direction. The Alkhalifah (2003) acoustic ORT assumption, which we call it the conventional acoustic assumption, has four important aspects: 1. It reduces the number of required model parameters from nine to six; 2 . It simplifies the governing equations required for seismic data processing and modelling; 3 . It is reasonably accurate (compared to the elastic case); 4. It decays the aforementioned $\mathrm{S}$-waves by reducing their propagation velocities, yet do not stop them, therefore, one of the main objectives of an acoustic assumption is not completely achieved in the conventional approach.

The first three characteristics of the conventional acoustic assumption resulted in widespread applications of it for ORT and TI media in most stages of seismic data processing, modelling, and parameter estimation. Examples of applications are in normal moveout correction (e.g. Abedi et al. 2019a), coherent noise attenuation (e.g. Abedi et al. 2019b), reverse time migration (e.g. Zhang \& Zhang 2011), ray tracing (e.g. Stovas et al. 2016; Waheed et al. 2015), wave propagation simulation (e.g. Song \& Alkhalifah 2013; Abedi et al. 2019c), fullwaveform inversion (e.g. Alkhalifah et al. 2016; Masmoudi \& Alkhalifah 2018), and any method that requires the approximation of P-wave kinematics (e.g. Stovas 2015; Stovas 2018).

On the other hand, many research efforts have been made to solve the problem of the presence of relatively strong S-waves artefacts that occur after the conventional acoustic assumption. For ORT media, several methods are proposed to mitigate the S-wave artefacts, or to eliminate them in specific modelling methods (Ibanez-Jacome et al. 2013; Song \& Alkhalifah 2013; Xu \& Liu 2018); these methods still use the conventional acoustic assumption. Recently, a new acoustic assumption that provides pure P wavefield for TI media is proposed by $\mathrm{Xu}$ et al. (2020). Following their method, in this study, we propose a new acoustic assumption for ORT media that can stop the S-wave artefacts from propagating on the cost of complicating the governing equations instead of simplifying them. Noticing the added algebraic complexities, we also propose simple phase velocity, and slowness approximations designed for the new acoustic ORT media; then study both the new acoustic assumption and the new approximate equations by numerical experiments.

\section{THEORY}

\section{New acoustic orthorhombic assumption}

An orthorhombic (ORT) medium is characterized by three mutually orthogonal symmetry planes, and described by nine independent density-normalized stiffness coefficients $\left(c_{i j}\right)$. To have similar parameters to those defined by Thomsen (1986) for TI media with a vertical axis 
of symmetry (VTI), Tsvankin (1997) rearranged the stiffness coefficient of ORT media into $v_{P 0}, v_{S 0}$ ( vertical velocities of $\mathrm{P}$-wave and the $\mathrm{S}$-wave that is polarized in $x_{1}$ direction), $\varepsilon^{(1)}$, $\varepsilon^{(2)}, \gamma^{(1)}, \gamma^{(2)}$ (anisotropy parameters defined in the vertical symmetry planes), and $\delta^{(1)}, \delta^{(2)}$, $\delta^{(3)}$ (anisotropy parameters defined in each symmetry plane). For the purpose of this study, we need the ORT parameters to be equivalently defined in all the three symmetry planes. Therefore, assuming that the intercepts of the symmetry planes coincide with the axes of the Cartesian coordinate, we define three axial $\mathrm{P}$-wave velocities, $v_{1}, v_{2}, v_{3}$, three axial $\mathrm{S}$-wave velocities that are polarized in each symmetry plane, $v_{S 1}, v_{S 2}, v_{S 3}$ (numbered with the axis normal to both propagation axis and polarization axis), and three eta parameters $\left(\eta_{1}, \eta_{2}, \eta_{3}\right)$ in each symmetry plane,

$$
\begin{aligned}
& v_{1}^{2}=c_{11}, v_{2}^{2}=c_{22}, v_{3}^{2}=c_{33}, \\
& v_{S 1}^{2}=c_{44}, v_{S 2}^{2}=c_{55}, v_{S 3}^{2}=c_{66}, \\
& \eta_{1}=\frac{c_{33}\left(c_{22}-c_{44}\right)}{2\left(c_{23}^{2}+c_{22} c_{44}+2 c_{23} c_{44}\right)}-\frac{1}{2}, \\
& \eta_{2}=\frac{c_{11}\left(c_{33}-c_{55}\right)}{2\left(c_{13}^{2}+c_{33} c_{55}+2 c_{13} c_{55}\right)}-\frac{1}{2}, \\
& \eta_{3}=\frac{c_{22}\left(c_{11}-c_{66}\right)}{2\left(c_{12}^{2}+c_{11} c_{66}+2 c_{12} c_{66}\right)}-\frac{1}{2} .
\end{aligned}
$$

Figure 1 shows how these parameters are defined with respect to the symmetry planes (e.g. in $x_{1}-x_{3}$ plane, which is identified by number 2 , we have $v_{1}, v_{3}$, which are horizontal and vertical P-wave velocities, $v_{S 2}$ and $\eta_{2}$ ). The medium is reduced to isotropic when $v_{1}=v_{2}=v_{3}$, $v_{S 1}=v_{S 2}=v_{S 3}$, and $\eta_{1}=\eta_{2}=\eta_{3}=0$. Figure 2(a) shows phase velocity surfaces, obtained as the solution to the characteristic equation (Appendix A) of the so-called Standard elastic ORT model of Schoenberg and Helbig (1997), given in Table 1. This figure shows one P- and two $\mathrm{S}$-wave velocity surfaces. In ORT media, the sensitivity of the P-wave kinematics to the parameters describing S-wave velocities along the axes is known to be low. Taking advantage of this weak sensitivity, the conventional acoustic assumption of Alkhalifah (2003) is obtained by setting $v_{S k}=0$, where the index $k=\{1,2,3\}$. Figure 2(b) shows phase velocity surfaces for the same medium as in Figure 2(a), but after the Alkhalifah (2003) acoustic assumption. The conventional acoustic assumption stops the S-waves along the three axes, yet the two S-waves still exist elsewhere as real positive solutions to the characteristic equation (Figure 2(d)). To address the problem of the remaining S-waves, we propose a new acoustic assumption for ORT media by zeroing the S-waves within the three symmetry planes.

For wave propagation in each symmetry plane of elastic ORT media, there is an S-wave that is polarized within the plane, and another that is polarized orthogonal to it. Here, we define the $v_{S k}$ parameters so that the phase velocity of the in-plane polarized S-waves, which are 
coupled with P-waves in each symmetry plane, go to zero. Therefore, instead of the simple zeroing of $v_{S k}$ parameters, in the new acoustic ORT assumption, the $v_{S k}$ parameters are obtained as functions of phase direction $\mathbf{n}$,

$v_{S k}^{2}(\mathbf{n})=-\frac{2 \eta_{k} n_{i}^{2} n_{j}^{2} v_{i}^{2} v_{j}^{2}}{n_{i}^{2}\left(n_{i}^{2}+n_{j}^{2}\right)\left(1+2 \eta_{k}\right) v_{i}^{2}+n_{j}^{2}\left(n_{i}^{2}+\left(1+2 \eta_{k}\right) n_{j}^{2}\right) v_{j}^{2}}$,

where $(i, j, k)$ are the indices with three possible combinations $(1,2,3),(2,3,1),(3,1,2)$. Note that $\mathbf{n}$ has two components in each symmetry plane. Equation (2) is analogous to equation 11 given in Xu et al. (2020) for VTI media. The phase velocity and slowness surfaces for the new acoustic ORT media are calculated in Appendix A, and Appendix B, respectively. Figure 2(c) shows the phase-velocity surfaces for the same medium as in Figure 2 (a), but after the new acoustic assumption. A small remnant of one S-wave velocity surface is visible (Figure 2(e)). Figure 3 shows the accuracy of the P-wave velocity surfaces in Figure 2(b) and (c), compared to Figure 2(a). Figure 3 also shows the accuracy comparison for another ORT model in Mah and Schmitt (2003), named as Model 1 (Table 1). The relative error surfaces for the new and the conventional acoustic assumption are similar.

\section{Comments}

1. The parameterization that we use (equation (1)) is suitable for acoustic media because only after an acoustic assumption the $\eta_{k}$ parameters define the degree of deviation from ellipsoidal slowness surface, hence, can be called anellipticity parameters. Appendix C shows the conversion from Tsvankin's (1997) parameters to the parameters in equation (1). Since in Tsvankin (1997), both parameters $\delta^{(1)}$ and $\delta^{(2)}$ are defined along the $x_{3}$ axis, we suggest to change the definition of $\delta^{(1)}$ to $\delta^{\left(1^{*}\right)}$ that is defined along the $x_{2}$ axis (as proposed in equation $\mathrm{C} 2$ ). Using the $\delta^{\left(1^{*}\right)}$, the elastic parameters are more concisely converted to the new acoustic parameters.

2. The conventional acoustic assumption results in an internal symmetry, which is not preserved in the new acoustic assumption. It can be checked through the Taylor series expansion of phase velocity squared along the two axes in a symmetry plane (Appendix D). In the conventional acoustic media, an interchange of $v_{i}$ and $v_{j}, n_{i}$ and $n_{j}$ does not change the kinematics in the symmetry plane; but, in the new acoustic assumption, such interchange slightly changes the kinematics, starting from the fourth-order derivative on one axis and sixth-order derivative on another. In the $x_{3}-x_{1}$ plane, the new and the conventional acoustic assumptions better match each other along the $x_{3}$ axis; in the $x_{1}-x_{2}$ plane, they better match along the $x_{1}$ axis, and in the $x_{2}-x_{3}$ plane they better match along the $x_{2}$ axis (Appendix D).

3. In conventional acoustic assumption, the $v_{S k}^{2}$ parameters (which are positive values in the elastic case) are set to zero. In the new acoustic assumption, the definition of $v_{S k}^{2}$ in equation (2) becomes nonpositive for $\eta_{k}>0$, and positive for $-0.5<\eta_{k}<0$. It means that the changes in $v_{S k}^{2}$ parameters can be less or greater compared to the conventional 
acoustic assumption depending on the $\eta_{k}$ parameter. Note that when $v_{S k}^{2}$ are negative the S-waves are better suppressed.

4. The characteristic equation of the new acoustic ORT media (in Appendix A) has three real roots, one of which is nonpositive for $\eta_{k}>0$; therefore, one S-wave is completely stopped. The second S-wave may have slow remnants or be completely stopped, depending on the model parameters (see the numerical analysis section).

\section{New phase velocity approximation}

The exact phase velocity in ORT media is analytically obtained by solving the characteristic equation, which is a cubic function of the phase velocity squared (Appendix A). However, the result is algebraically too complicated for some practical purposes. The algebraic complexity is increased after the new acoustic ORT assumption because the $v_{S k}^{2}$ parameters are replaced with the expression in equation (2). Accordingly, an algebraically simpler approximate equation can be useful in practice. To obtain such approximation we use the phase velocity expression in TI media (equation E1) and make a 3D expansion of it based on the equivalence between P-wave kinematics in the symmetry planes of ORT and TI media. We consider a 3D ellipsoidal background and add anelliptic terms from each symmetry plane to obtain a simple rational approximation for the phase velocity squared within the framework of the new acoustic ORT assumption,

$$
\begin{aligned}
v^{2}(\mathbf{n}) & \simeq e-\frac{2 \eta_{1} n_{2}^{2} n_{3}^{2}\left(n_{2}^{2}+n_{3}^{2}\right) v_{2}^{2} v_{3}^{2}}{\left(1+2 \eta_{1}\right)\left(n_{2}^{2}+n_{3}^{2}\right) e-2 \eta_{1} n_{2}^{2} n_{3}^{2} v_{3}^{2}}-\frac{2 \eta_{2} n_{1}^{2} n_{3}^{2}\left(n_{1}^{2}+n_{3}^{2}\right) v_{1}^{2} v_{3}^{2}}{\left(1+2 \eta_{2}\right)\left(n_{1}^{2}+n_{3}^{2}\right) e-2 \eta_{2} n_{1}^{2} n_{3}^{2} v_{1}^{2}} \\
& -\frac{2 \eta_{3} n_{1}^{2} n_{2}^{2}\left(n_{1}^{2}+n_{2}^{2}\right) v_{1}^{2} v_{2}^{2}}{\left(1+2 \eta_{3}\right)\left(n_{1}^{2}+n_{2}^{2}\right) e-2 \eta_{3} n_{1}^{2} n_{2}^{2} v_{2}^{2}},
\end{aligned}
$$

where $e=v_{1}^{2} n_{1}^{2}+v_{2}^{2} n_{2}^{2}+v_{3}^{2} n_{3}^{2}$ is the ellipsoidal part of the phase-velocity. Note that this approximation is different from the rational approximation in Abedi (2020) because, within the symmetry planes, equation (3) is reduced to the exact expression of phase velocity squared under the new acoustic assumption (Appendix E). Figure 4 shows the relative errors of the proposed phase velocity approximation (equation (3)) compared with the exact solution under the new acoustic assumption (equation A5). The equation (3) gives the new acoustic phase velocity along the symmetry planes $\left(n_{k}=0\right)$, and an approximation of it outside the symmetry planes of ORT media.

To have another phase velocity approximation for the new acoustic ORT media, one can modify the parameters of the generalized velocity approximation (GVA; Stovas and Fomel, 2019) as calculated in Appendix F. With three square roots in its functional form, the GVA (equation F1) is algebraically more complicated than the proposed rational approximation (equation (3)). Figure 4 shows the relative error of the original GVA (Stovas and Fomel, 2019), and the modified GVA that is tailored for the new acoustic assumption (Appendix F); they are not exact in all directions along the symmetry planes. 
The potential application of the proposed phase-velocity approximation is in wavefield modelling. Converting equation (3) into a dispersion relation in the frequency domain, it can be used in wave extrapolation by the low-rank approximation (Fomel et al. 2013) as is shown in Song and Alkhalifah (2013), Sripanich and Fomel (2015), and Abedi et al. (2019c). The dispersion relation, which relates the wavenumber vector of a wave (k) to its temporal frequency $(\phi)$, is obtained after replacing $v^{2}(\mathbf{n})$ in equation 3 with the phase operator $\phi^{2}(\mathbf{n})$, and $n_{j}$ with the components of wavenumber $k_{j}$ (Abedi et al. 2019c).

\section{New slowness approximation}

The exact slowness surface in ORT media (equation B1) is obtained from the determinant of the Christoffel matrix that is written in the slowness domain. To address the issue of algebraic complexity of the exact slowness and eikonal equations under the new acoustic assumption (Appendix B), we propose a new approximation for slowness surface, then convert it to an eikonal equation. Using a similar method for the proposed approximation in equation (3), we obtain an approximate slowness surface by a 3D expansion of the slowness equation in each symmetry plane of ORT media. The proposed slowness surface reads,

$$
\begin{aligned}
& 2\left(\frac{\eta_{3} p_{1}^{2} p_{2}^{2} v_{1}^{2} v_{2}^{2}}{1+2 \eta_{3}}+\frac{\eta_{2} p_{3}^{2} p_{1}^{2} v_{1}^{2} v_{3}^{2}}{1+2 \eta_{2}}+\frac{\eta_{1} p_{2}^{2} p_{3}^{2} v_{2}^{2} v_{3}^{2}}{1+2 \eta_{1}}\right) \\
& -\left(e_{p}-1\right)\left(e_{p}-\frac{2 \eta_{2} p_{1}^{2} p_{3}^{2} v_{1}^{2}}{\left(1+2 \eta_{2}\right)\left(p_{1}^{2}+p_{3}^{2}\right)}-\frac{2 \eta_{3} p_{1}^{2} p_{2}^{2} v_{2}^{2}}{\left(1+2 \eta_{3}\right)\left(p_{1}^{2}+p_{2}^{2}\right)}-\frac{2 \eta_{1} p_{2}^{2} p_{3}^{2} v_{3}^{2}}{\left(1+2 \eta_{1}\right)\left(p_{2}^{2}+p_{3}^{2}\right)}\right)=0
\end{aligned}
$$

where $e_{p}=v_{1}^{2} p_{1}^{2}+v_{2}^{2} p_{2}{ }^{2}+v_{3}^{2} p_{3}^{2}$, and $p_{j}$ are the components of slowness. Equation (4) is a biquartic equation in terms of $p_{3}$. Figure 5 shows the relative errors of the proposed slowness approximation (equation (4)) compared with the exact solution under the new acoustic assumption (equation B1). This figure shows the errors in $|p|=\sqrt{p_{1}^{2}+p_{2}^{2}+p_{3}^{2}}$. Equation (4) gives the new acoustic slowness along the symmetry planes of ORT media and an approximation of it out of the planes.

Next, replacing, $p_{1}=\frac{d t}{d x}, p_{2}=\frac{d t}{d y}, p_{3}=\frac{d t}{d z}$, equation (4) is converted to a first-order partial differential equation (PDE), which is known as the eikonal equation, for the new acoustic ORT media. Equation (4) is much simpler than the exact eikonal equation under the new acoustic assumption (Appendix B). Equation (4) is reduced to the exact slowness surface (and eikonal equation after the aforementioned conversion) in 2D acoustic TI media, setting one component of slowness $p_{j}$ to zero (equation E2). 


\section{NUMERICAL ANALYSIS}

A numerical experiment is arranged to study the properties and accuracy of the new acoustic assumption in multiple ORT models. We use a set of models defined with parameters $v_{1}, v_{2}, v_{3} \in\{2.5,3,3.5\} \mathrm{km} / \mathrm{s}, \eta_{1}, \eta_{2}, \eta_{3} \in\{0.1,0.2,0.3\}$, and $v_{S 1}=1, v_{S 2}=1.15, v_{S 3}=1.3, \mathrm{~km} / \mathrm{s}$; there are six varying elastic parameters (and three fixed), where each varying-parameter accepts any of the three values, forming 729 ORT models (Table 1).

First, we check the discriminant under the new acoustic assumption (equation (A4)) for the presence of three real roots of the characteristic equation. As Figure 6(a) shows, the discriminant is nonnegative, therefore, the trigonometric solution (equation (A5)) is the correct approach for calculating the exact phase velocities under the new acoustic assumption. Second, we calculate the exact $\mathrm{P}$-wave phase velocities under the new and the conventional acoustic assumptions and compare them with the original elastic values. Figure 6(b) shows the maximum of absolute relative errors. The new acoustic assumption is generally less accurate than the conventional one, but maintains within the same orders of errors. Third, we evaluate the success of the new acoustic assumption in avoiding the S-wave artefacts, which is its main objective. Figure 6(c) and (d) show the maximum value of S-waves phase velocities squared in acoustic ORT media, sorted by their values in the conventional acoustic assumption. As Figure 6(c) shows, the maximum value of the $\mathrm{S} 1$ phase velocity squared is nonpositive after the new acoustic assumption, therefore, no S1 is propagated. Figure 6(d) shows that the maximum value of S2 phase velocity is negative in some models which means no S2 is propagated either; however, it shows positive values for other models, which means S2 propagates but with a smaller velocity than the conventional acoustic assumption. For example, in a model with $v_{1}=3, v_{2}=3.5, v_{3}=2.5, \mathrm{~km} / \mathrm{s}, \eta_{1}=0.3, \eta_{2}=0.1, \eta_{3}=0.2$, both S-waves are stopped after the new acoustic assumption. In the same model but $\eta_{3}=0.1$, S2 propagates but with a decreased maximum velocity compared to the conventional method. Figure 1 shows a model of the latter case. Note that both S-waves are always stopped within the symmetry planes and in their vicinities.

The same set of ORT models introduced in Table 1 are used to evaluate the accuracy of the proposed rational phase velocity approximation (equation (3)). Figure 7(a) shows the maximum of absolute relative errors of the proposed equation (3) and the modified GVA for the new acoustic assumption (Appendix F), when compared with the exact P-wave velocity under the new acoustic assumption (equation (A5)). The proposed rational phase-velocity approximation is more accurate than the modified GVA in all the models.

Although the equation (3) is proposed to approximate the P-wave phase-velocity under the new acoustic assumption, due to the comparable accuracy of the new and the conventional acoustic ORT media, it can also be used for conventional acoustic media. Figure 7(b) replicates Figure 7(a) for the same equation (3), but the original GVA (equation 32 in Stovas and Fomel, 2019) when compared with the exact P-wave velocity under the conventional acoustic assumption. The maximum error of the proposed rational phase velocity approximation is less than that of the GVA in most of the models. 
A phase-velocity or slowness approximation is used in wavefield modelling and ray tracing. Here we use the proposed approximation in slowness domain (equation (4)) in a simple ray-tracing scheme in the ORT Model 1 with positive anellipticity parameters. Figure 8(a) shows the traced rays in a 3D acquisition pattern with fixed source and receiver locations. Figure $8(\mathrm{~b})$ shows the modelled P-wave shot gather which is obtained by convolving a Ricker wavelet with traveltimes calculated by ray tracing. Figure 8(c) shows the absolute value of relative errors of the calculated traveltimes compared with the exact traveltimes under the acoustic assumption. The calculated traveltime using equation (4) is accurate enough to be used instead of the more complicated exact equation under the new acoustic assumption.

\section{DISCUSSION}

The proposed method is an assumption that is made on wave propagation, trying to stop Swaves from being propagated. To apply the new acoustic assumption, we insert the $c_{i j}$ parameters into equation 1 to reach the new notation, then change the definition of the $v_{S k}$ parameters by the expression given in equation 2 . This method is applicable for general ORT media and all the special cases. Vertically fractured TI media is a special ORT model that is defined by eight independent elastic parameters and a constraint (Bakulin, 2000). After the conventional acoustic assumption, the constraint remains valid and the number of independent parameters is reduced to five. However, after the new acoustic assumption, six parameters remain independent.

In the introduction section, we count four characteristics for the conventional acoustic assumption in general ORT media. Regarding them, our new acoustic assumption keeps the reduction of the required model parameters, maintains the same level of accuracy, and mitigates the S-wave artefacts problem, but deteriorates the complexities of the governing equations in ORT media. For example, the eikonal equation for the new acoustic ORT media has a total degree of 28. Although an eikonal equation is a first-order PDE to be solved numerically, it will still be complicated to be solved for a degree of 28 . To mitigate this problem, we also proposed an approximate eikonal equation for the new acoustic ORT media. The total degree of the new approximate acoustic eikonal equation is 10 .

\section{CONCLUSION}

To stop S-wave artefacts from propagating, we propose a new acoustic assumption for orthorhombic media. First, we employ a new parameterization for elastic orthorhombic media, using three axial P-wave velocities, three axial S-wave velocities, and three in-plane eta parameters. Then, instead of zeroing out the S-wave velocities on axes, which is the conventional acoustic assumption, we zero out the S-wave velocities within the symmetry planes of orthorhombic media. Based on the numerical experiments on multiple orthorhombic models, the new acoustic assumption completely stops one S-wave, may stop or leave small remnants of the second S-wave, and keeps the P-wave kinematic properties reasonably accurate. The cost of the mitigation of the S-wave artefacts is the added complexity to the phase velocity, slowness, and eikonal equations. We address this issue by proposing simple and 
rational phase-velocity, and slowness approximations for the new acoustic orthorhombic media. They reduce to exact equations within the symmetry planes. Based on our numerical studies, the proposed phase-velocity approximation is more accurate than generalized velocity approximation. The proposed slowness approximation is converted to an approximate eikonal equation and is used in a simple ray tracing in a standard orthorhombic model, which shows its applicability.

\section{ACKNOWLEDGEMENTS}

We would like to acknowledge the journal reviewers, Igor Ravve, and Subhashis Mallick for their insightful comments. The first author received funding from the European POCTEFA Project PIXIL (EFA362/19), the grant Artificial Intelligence in BCAM number EXP. 2019/00432, and the Project of the Spanish Ministry of Science and Innovation with reference PID2019-108111RB-I00 (FEDER/AEI). The second author received funding from the GAMES project at NTNU.

\section{REFERENCES}

Abedi, M. M., 2020. Rational approximation of P-wave kinematics: Part 2 - Orthorhombic media, Geophysics, 85(5), C175-C186.

Abedi, M. M., Riahi, M. A. \& Stovas, A., 2019a. Three-parameter normal moveout correction in layered anisotropic media: A stretch-free approach, Geophysics, 84(3), C129-C142.

Abedi, M. M., Riahi, M. A. \& Stovas, A., 2019b. Three-parameter Radon transform in layered transversely isotropic media, Geophysical Prospecting, 67, 395-407. doi:10.1111/13652478.12731

Abedi, M.M., Stovas, A. \& Ivanov, Y., 2019c. Acoustic wave propagation in orthorhombic media: phase velocity, group velocity, and moveout approximations, Geophysics, 84(6), C269-C279.

Alkhalifah, T. 1998. Acoustic approximations for seismic processing in transversely isotropic media, Geophysics, 63, 623-631.

Alkhalifah, T., 2003. An acoustic wave equation for orthorhombic anisotropy, Geophysics, 68 , 1169-1172, doi: 10.1190/1.1598109.

Alkhalifah, T., Masmoudi, N. \& Oh, J.W., 2016. A recipe for practical full-waveform inversion in orthorhombic anisotropy, The Leading Edge, 35(12), 1076-1083.

Bakulin, A., Grechka, V. \& I. Tsvankin, 2000. Estimation of fracture parameters for reflection seismic data; Part II, Fractured models with orthorhombic symmetry, Geophysics, 65, 18031817.

Fomel, S., L. Ying, \& X. Song, 2013. Seismic wave extrapolation using lowrank symbol approximation, Geophysical Prospecting, 61, 526-536. doi: 10.1111/j.13652478.2012.01064.x.

Ibanez-Jacome, W., Alkhalifah, T. \& Waheed, U. B., 2013. Effective orthorhombic anisotropic models for wavefield extrapolation, Geophysical Journal International, 198, 1653-1661, doi: $10.1093 /$ gji/ggu229. 
Mah, M. \& Schmitt, D.R., 2003. Determination of the complete elastic stiffnesses from ultrasonic phase velocity measurements, Journal of Geophysical Research: Solid Earth, 108(B1), ECV-6.

Masmoudi, N. \& Alkhalifah, T., 2018. Full-waveform inversion in acoustic orthorhombic media and application to a North Sea data set, Geophysics, 83(5), pp.C179-C193.

Schoenberg, M., \& K. Helbig, 1997. Orthorhombic media: Modeling elastic wave behavior in a vertically fractured earth, Geophysics, 62, 1954-1974, doi: 10.1190/1.1444297.

Song, X., \& T. Alkhalifah, 2013. Modeling of pseudoacoustic P-waves in orthorhombic media with a low-rank approximation, Geophysics, 78(4), C33-C40, doi: 10.1190/geo20120144.1.

Sripanich, Y.\& Fomel, S., 2015. On anelliptic approximations for qP velocities in transversely isotropic \& orthorhombic media, Geophysics, 80(5), C89-C105.

Stovas, A., 2015. Azimuthally dependent kinematic properties of orthorhombic media, Geophysics, 80(6), C107-C122.

Stovas, A., 2018. Geometrical spreading in orthorhombic media, Geophysics, 83 (1), C61-C73.

Stovas, A. \& Fomel, S., 2019. Generalized velocity approximation, Geophysics, 84 (1), C27C40.

Stovas, A., Masmoudi, N., \& T.Alkhalifah, 2016. Application of perturbation theory for Pwave eikonal equation in orthorhombic media, Geophysics, 81(6), C309-C317.

Thomsen, L., 1986, Weak elastic anisotropy, Geophysics, 51(10), 1954-1966.

Tsvankin, I., 1997. Anisotropic parameters and P-wave velocity for orthorhombic media: Geophysics, 62, 1292-1309. doi: 10.1190/1.1444231.

Waheed, U.B., Yarman, C.E. \& Flagg, G., 2015. An iterative, fast-sweeping-based eikonal solver for 3D tilted anisotropic media, Geophysics, 80(3), C49-C58.

$\mathrm{Xu}, \mathrm{S} . \&$ Liu, Y., 2018. Effective modeling and reverse-time migration for novel pure acoustic wave in arbitrary orthorhombic anisotropic media, Journal of Applied Geophysics, 150, 126-143.

Xu, S., Stovas, A., Alkhalifah, T. \& Mikada, H., 2020. New acoustic approximation for the transversely isotropic media with a vertical symmetry axis, Geophysics, 85(1), 1-58.

Zhang, H. \& Zhang, Y., 2011. Reverse time migration in vertical and tilted orthorhombic media, SEG Technical Program Expanded Abstracts, 185-189. 


\section{APPENDIX A}

\section{EXACT PHASE VELOCITIES IN ORTHORHOMBIC MEDIA}

The exact phase velocity expression in orthorhombic media is obtained as a solution of the characteristic equation, which is obtained from the determinant of the Christoffel matrix. The characteristic equation reads,

$v^{6}+b v^{4}+c v^{2}+d=0$,

with,

$$
\begin{aligned}
& b=-\left(G_{11}+G_{22}+G_{33}\right), \\
& c=G_{11} G_{22}+G_{11} G_{33}+G_{22} G_{33}-\left(G_{12}^{2}+G_{13}^{2}+G_{23}^{2}\right), \\
& d=G_{11} G_{23}^{2}+G_{22} G_{13}^{2}+G_{33} G_{12}^{2}-G_{11} G_{22} G_{33}-2 G_{12} G_{13} G_{23} .
\end{aligned}
$$

where $G_{i j}$ are the elements of the Christoffel matrix,

$$
\begin{aligned}
& G_{11}=v_{1}^{2} n_{1}^{2}+v_{S 3}^{2} n_{2}^{2}+v_{S 2}^{2} n_{3}^{2}, \\
& G_{22}=v_{S 3}^{2} n_{1}^{2}+v_{2}^{2} n_{2}^{2}+v_{S 1}^{2} n_{3}^{2}, \\
& G_{33}=v_{S 2}^{2} n_{1}^{2}+v_{S 1}^{2} n_{2}^{2}+v_{3}^{2} n_{3}^{2}, \\
& G_{12}=\sqrt{\frac{\left(v_{2}^{2}-\left(1+2 \eta_{3}\right) v_{S 3}^{2}\right)\left(v_{1}^{2}-v_{S 3}^{2}\right)}{1+2 \eta_{3}}}, \\
& G_{13}=\sqrt{\frac{\left(v_{1}^{2}-\left(1+2 \eta_{2}\right) v_{S 2}^{2}\right)\left(v_{3}^{2}-v_{S 2}^{2}\right)}{1+2 \eta_{2}}}, \\
& G_{23}=\sqrt{\frac{\left(v_{3}^{2}-\left(1+2 \eta_{1}\right) v_{S 1}^{2}\right)\left(v_{2}^{2}-v_{S 1}^{2}\right)}{1+2 \eta_{1}}} .
\end{aligned}
$$

The $v_{S k}^{2}$ parameters are replaced with equation (2), to reach the new acoustic assumption. The characteristic equation is a cubic polynomial equation in terms of $v^{2}$. The discriminant of equation (A1),

$$
\Delta=18 b c d-4 b^{3} d+b^{2} c^{2}-4 c^{3}-27 d^{2},
$$

determines whether all the roots are real or not; if $\Delta \geq 0$, equation (A1) has three real roots. The three real roots are obtained as

$$
v^{2}(\mathbf{n})=2 \sqrt{-\frac{p}{3}} \cos \left[\frac{1}{3} \arccos \left[\frac{3 q}{2 p} \sqrt{-\frac{3}{p}}\right]+\frac{2 m \pi}{3}\right]-\frac{b}{3}, \quad m=0,1,2
$$

where,

$$
p=\frac{3 c-b^{2}}{3}, q=\frac{2 b^{3}-9 b c+27 d}{27} .
$$

The roots correspond to the desired $\mathrm{P}$-wave, S1 artefact, and S2 artefact, respectively. We call the resultant P-wave velocity from equation (A5) the exact phase velocity under the new acoustic assumption to defer it from equation (3) which gives an approximation of it. 


\section{APPENDIX B}

\section{SLOWNESS SURFACE IN ORTHORHOMBIC MEDIA}

The slowness surface in orthorhombic media is calculated from the Christoffel equation as,

$$
\begin{aligned}
& \sum_{i} p_{j}^{2} p_{k}^{2}\left(p_{k}^{2} v_{S j}^{2}+p_{j}^{2} v_{S k}^{2}+p_{i}^{2} v_{i}^{2}-1\right)\left(v_{j}^{2}-v_{S i}^{2}\right)\left(v_{S i}^{2}-\frac{v_{k}^{2}}{1+2 \eta_{i}}\right)+ \\
& 2 \prod_{i} \sqrt{p_{i}^{4}\left(v_{S i}^{2}-v_{j}^{2}\right)\left(v_{S i}^{2}-\frac{v_{k}^{2}}{1+2 \eta_{i}}\right)}+\prod_{i}\left(p_{k}^{2} v_{S j}^{2}+p_{j}^{2} v_{k}^{2}+p_{i}^{2} v_{i}^{2}-1\right)=0
\end{aligned}
$$

where $(i, j, k)$ are the indices; in the new acoustic assumption,

$$
v_{S k}^{2}=-\frac{2 \eta_{k} p_{i}^{2} p_{j}^{2} v_{i}^{2} v_{j}^{2}}{p_{i}^{2}\left(p_{i}^{2}+p_{j}^{2}\right)\left(1+2 \eta_{k}\right) v_{i}^{2}+p_{j}^{2}\left(p_{i}^{2}+\left(1+2 \eta_{k}\right) p_{j}^{2}\right) v_{j}^{2}},
$$

The definition in equation (B2) increases the degree of equation (B1) in terms of slowness components.

\section{APPENDIX C}

\section{CONVERSION FROM TSVANKIN'S TO THE NEW ACOUSTIC PARAMETERS}

Here we obtain relations that are used to convert the Tsvankin (1997) parameters for ORT media to the new acoustic ORT assumption. We have,

$$
\begin{aligned}
& v_{P 0}^{2}=v_{3}^{2}, v_{S 0}^{2}=v_{S 2}^{2}, \\
& \varepsilon^{(2)}=\frac{1}{2}\left(\frac{v_{1}^{2}}{v_{3}^{2}}-1\right), \gamma^{(2)}=\frac{1}{2}\left(\frac{v_{S 3}^{2}}{v_{S 1}^{2}}-1\right), \\
& \varepsilon^{(1)}=\frac{1}{2}\left(\frac{v_{2}^{2}}{v_{3}^{2}}-1\right), \gamma^{(1)}=\frac{1}{2}\left(\frac{v_{S 3}^{2}}{v_{S 2}^{2}}-1\right), \\
& \delta^{(3)}=\frac{1}{2}\left(\frac{v_{2}^{2}}{\left(1+2 \eta_{3}\right) v_{1}^{2}}-1\right), \delta^{(2)}=\frac{1}{2}\left(\frac{v_{1}^{2}}{\left(1+2 \eta_{2}\right) v_{3}^{2}}-1\right), \\
& \delta^{(1)}=\frac{1}{2}\left(\frac{v_{2}^{2}}{v_{3}^{2}}-\frac{2 \eta_{1}\left(v_{2}^{2}-v_{S 1}^{2}\right)}{\left(1+2 \eta_{1}\right)\left(v_{3}^{2}-v_{S 1}^{2}\right)}-1\right) .
\end{aligned}
$$

The parameters $v_{S 0}^{2}, \gamma^{(1)}, \gamma^{(2)}$ are dependent on $v_{S k}^{2}$, as expected. However, the parameter $\delta^{(1)}$ also depends on $v_{S k}^{2}$, and remains a function of phase direction after the new acoustic assumption; it is undesirable. The reason behind this problem is that both parameters $\delta^{(1)}$ and $\delta^{(2)}$ are defined along the $x_{3}$ axis; alternately, defining a $\delta^{\left(1^{*}\right)}$ parameter along the $x_{2}$ axis,

$$
\delta^{\left(1^{*}\right)}=\frac{\left(c_{23}+c_{44}\right)^{2}-\left(c_{22}-c_{44}\right)^{2}}{2 c_{22}\left(c_{22}-c_{44}\right)},
$$


the conversion to the new acoustic parameters takes the more concise form,

$$
\delta^{\left(1^{*}\right)}=\frac{1}{2}\left(\frac{v_{3}^{2}}{\left(1+2 \eta_{1}\right) v_{2}^{2}}-1\right) .
$$

\section{APPENDIX D}

\section{SERIES OF PHASE VELOCITY IN SYMMETRY PLANES}

We calculate the series expansion of exact phase velocity squared under the conventional and the new acoustic assumptions and compare them with the elastic case. For the conventional acoustic assumption, the series in $x_{3}-x_{1}$ plane read,

$$
\begin{aligned}
& v^{2}\left(n_{1} \rightarrow 0,0, n_{3}\right) \approx v_{3}^{2}+\left(\frac{v_{1}^{2}}{1+2 \eta_{2}}-v_{3}^{2}\right) n_{1}^{2}+\frac{2 \eta_{2} v_{1}^{4} n_{1}^{4}}{v_{3}^{2}\left(1+2 \eta_{2}\right)^{2}}+2 \eta_{2} v_{1}^{4} \frac{\left(2 \eta_{2}-1\right) v_{1}^{2}+\left(1+2 \eta_{2}\right) v_{3}^{2}}{\left(1+2 \eta_{2}\right)^{3} v_{3}^{4}} n_{1}^{6}+\ldots \\
& v^{2}\left(n_{1}, 0, n_{3} \rightarrow 0\right) \approx v_{1}^{2}+\left(\frac{v_{3}^{2}}{1+2 \eta_{2}}-v_{1}^{2}\right) n_{3}^{2}+\frac{2 \eta_{2} v_{3}^{4} n_{3}^{4}}{v_{1}^{2}\left(1+2 \eta_{2}\right)^{2}}+2 \eta_{2} v_{3}^{4} \frac{\left(2 \eta_{2}-1\right) v_{3}^{2}+\left(1+2 \eta_{2}\right) v_{1}^{2}}{\left(1+2 \eta_{2}\right)^{3} v_{1}^{4}} n_{3}^{6}+\ldots
\end{aligned}
$$

For the new acoustic assumption, the series in $x_{3}-x_{1}$ plane read,

$$
\begin{aligned}
& v^{2}\left(n_{1} \rightarrow 0,0, n_{3}\right) \approx v_{3}^{2}+\left(\frac{v_{1}^{2}}{1+2 \eta_{2}}-v_{3}^{2}\right) n_{1}^{2}+\frac{2 \eta_{2} v_{1}^{4} n_{1}^{4}}{v_{3}^{2}\left(1+2 \eta_{2}\right)^{2}}+2 \eta_{2} v_{1}^{4} \frac{v_{3}^{2}\left(1+2 \eta_{2}\right)^{2}-v_{1}^{2}}{\left(1+2 \eta_{2}\right)^{3} v_{3}^{4}} n_{1}^{6}+\ldots \\
& v^{2}\left(n_{1}, 0, n_{3} \rightarrow 0\right) \approx v_{1}^{2}+\left(\frac{v_{3}^{2}}{1+2 \eta_{2}}-v_{1}^{2}\right) n_{3}^{2}+\frac{2 \eta_{2} v_{3}^{2}\left(v_{3}^{2}+2 \eta_{2}\left(v_{3}^{2}-v_{1}^{2}\right)\right) n_{3}^{4}}{v_{1}^{2}\left(1+2 \eta_{2}\right)^{2}}+\ldots
\end{aligned}
$$

The terms in D2 match those in D1 up to the third term along $x_{3}$, and up to the second term along $x_{1}$ axis. This non-symmetry is inherited from the elastic case where it starts earlier in terms of series expansion,

$$
\begin{aligned}
& v^{2}\left(n_{1} \rightarrow 0,0, n_{3}\right) \approx v_{3}^{2}+\left(\frac{v_{1}^{2}}{1+2 \eta_{2}}-v_{3}^{2}\right) n_{1}^{2}+\frac{2 \eta_{2} v_{1}^{2}\left(\left(1+2 \eta_{2}\right) v_{S 2}^{2}-v_{1}^{2}\right) n_{1}^{4}}{\left(1+2 \eta_{2}\right)^{2}\left(v_{S 2}^{2}-v_{3}^{2}\right)}+\ldots \\
& v^{2}\left(n_{1}, 0, n_{3} \rightarrow 0\right) \approx v_{1}^{2}+\frac{v_{1}^{2} v_{3}^{2}-\left(1+2 \eta_{2}\right) v_{1}^{4}+v_{S 2}^{2}\left(\left(1+4 \eta_{2}\right) v_{1}^{2}-\left(1+2 \eta_{2}\right) v_{3}^{2}\right)}{\left(1+2 \eta_{2}\right)\left(v_{1}^{2}-v_{S 2}^{2}\right)} n_{3}^{2}+\ldots
\end{aligned}
$$

Therefore, the new acoustic assumption is more symmetrical than the elastic case.

\section{APPENDIX E}

\section{VTI MEDIA UNDER THE NEW ACOUSTIC ASSUMPTION}

Defining $v_{S 0}$ in transversely isotropic (TI) media through equation (2), the P-wave phase velocity under the new acoustic assumption is calculated from the Christoffel equation as, 


$$
v^{2}(\mathbf{n})=n_{1}^{2} v_{1}^{2}+n_{3}^{2} v_{3}^{2}-\frac{2 \eta n_{1}^{2} n_{3}^{2} v_{1}^{2} v_{3}^{2}}{\left(1+2 \eta n_{1}^{2}\right) n_{1}^{2} v_{1}^{2}+(1+2 \eta) n_{3}^{2} v_{3}^{2}}
$$

where $\mathbf{n}=(\sin \theta, \cos \theta), \theta$ is the phase angle from vertical, $v_{3}$ is the vertical, and $v_{1}$ is the horizontal velocities. Unlike the exact phase velocity expression in the conventional acoustic TI media, equation E1 is rational. Writing the Christoffel equation of a TI medium in slowness domain, its determinant gives the slowness surface as follows,

$$
\begin{aligned}
& v_{3}^{4} p_{3}^{6}+v_{3}^{2}\left(p_{1}^{2}\left(\frac{2 v_{1}^{2}}{1+2 \eta}+v_{3}^{2}\right)-1\right) p_{3}^{4}+ \\
& \left(v_{3}^{2}\left(p_{1}^{2} v_{1}^{2}-1\right)+v_{1}^{2} \frac{p_{1}^{2}\left(v_{1}^{2}+v_{3}^{2}\right)-1}{1+2 \eta}\right) p_{1}^{2} p_{3}^{2}+v_{1}^{4} p_{1}^{6}-v_{1}^{2} p_{1}^{4}=0
\end{aligned}
$$

where $p_{1}$ and $p_{3}$ are horizontal and vertical components of slowness. Equation (E2) is a bicubic equation in terms of each slowness components. Next, replacing, $p_{1}=\frac{d t}{d x}, p_{2}=\frac{d t}{d y}, p_{3}=\frac{d t}{d z}$, in the above equation, it is converted to a first-order PDE, known as the eikonal equation for the new acoustic VTI media. The total degree of equation (E2) is 6.

\section{APPENDIX F}

\section{ANOTHER PHASE VELOCITY APPROXIMATION FOR THE NEW ACOUSTIC ORTHORHOMBIC MEDIA}

Generalized velocity approximation (GVA; Stovas and Fomel, 2019) proposes flexible functions that can be used to fit in different media. The GVA for phase velocity in homogeneous media reads,

$$
v^{2}(\mathbf{n}) \simeq\left(1-\lambda_{1}-\lambda_{2}-\lambda_{3}\right) e+\lambda_{1} \sqrt{e^{2}+b_{1} n_{2}^{2} n_{3}^{2}}+\lambda_{2} \sqrt{e^{2}+b_{2} n_{1}^{2} n_{3}^{2}}+\lambda_{3} \sqrt{e^{2}+b_{3} n_{1}^{2} n_{2}^{2}}
$$

where $e$ is the ellipsoidal part, and $\lambda_{k}$ and $b_{k}$ are the equation parameters that should be defined according to the medium. Calculating the parameters of (F1) from the new acoustic ORT kinematics,

$$
\begin{aligned}
& \lambda_{k}=\frac{\left(1+2 \eta_{k}\right) v_{i}^{2}}{\left(1+\eta_{k}\right) v_{j}^{2}+\left(1+2 \eta_{k}\right) v_{i}^{2}}, \\
& \mathrm{~b}_{k}=-4 \eta_{k} v_{j}^{2} \frac{\left(1+\eta_{k}\right) v_{j}^{2}+\left(1+2 \eta_{k}\right) v_{i}^{2}}{\left(1+2 \eta_{k}\right)^{2}},
\end{aligned}
$$

The $v_{i}, v_{j}$, and $\eta_{k}$ are model parameters in each symmetry plane. The modified definitions in (F2) fits the GVA in (F1) to exact phase velocity under the new acoustic assumption. It is fitted 
to the new acoustic phase velocities at $n_{j}^{2}=n_{k}^{2}=1 / 2$, and to the new acoustic phase velocities and their second-order derivative along $n_{k}^{2}=1$.

Table 1. Orthorhombic model parameters that are used to evaluate the proposed acoustic assumption, and the proposed approximate equations. The axial P-wave $\left(v_{k}\right)$, and S-wave velocities $\left(v_{S k}\right)$ are in $\mathrm{km} / \mathrm{s}$.

\begin{tabular}{cccccccccc}
\hline & $v_{1}$ & $v_{2}$ & $v_{3}$ & $v_{S 1}$ & $v_{S 2}$ & $v_{S 3}$ & $\eta_{1}$ & $\eta_{2}$ & $\eta_{3}$ \\
\hline Standard & 3 & 3.14 & 2.44 & 1.41 & 1.26 & 1.48 & 0.16 & 0.4 & 0.19 \\
& & & & & & & & & \\
\hline Model 1 & 3.99 & 3.94 & 3.33 & 1.84 & 1.73 & 1.95 & -0.044 & 0.035 & 0.077 \\
& & & & & & & & & \\
\hline $\begin{array}{c}\text { Multiple } \\
\text { Models }\end{array}$ & $2.5,3$, & $\begin{array}{c}2.5,3 \\
3.5\end{array}$ & $2.5,3$, & 1 & 1.15 & 1.3 & $0.1,0.2$, & $0.1,0.2$, & $0.1,0.2$, \\
\hline
\end{tabular}

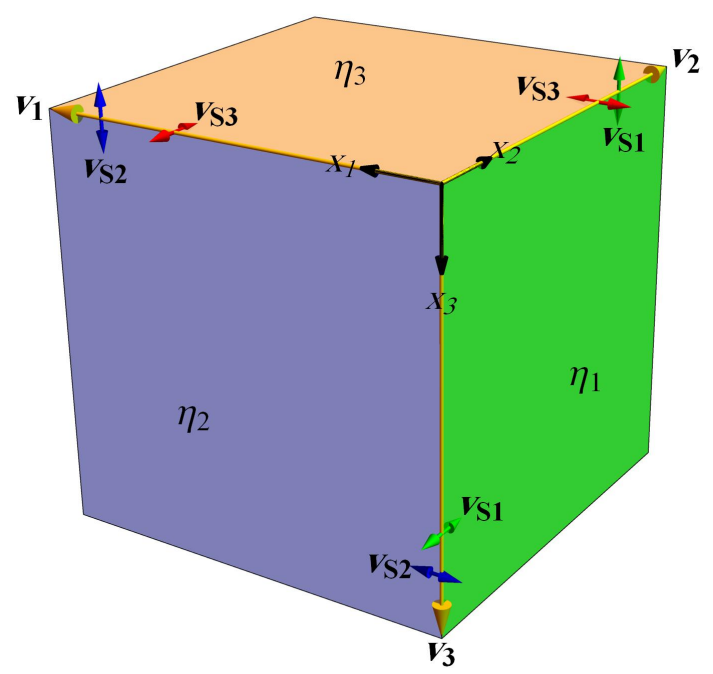

Figure 1. The definition of orthorhombic parameters in equation (1) with respect to the Cartesian coordinates. The double-headed arrows show the direction of polarization for $v_{S}$ parameters. Note the numbering of $v_{S}$ and $\eta$ parameters, based on the normal axis. 
a)

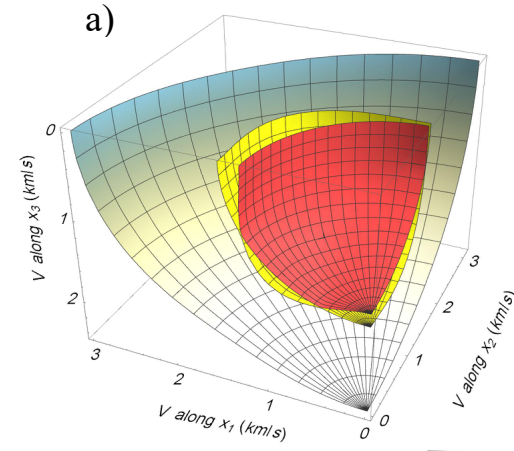

d) b)

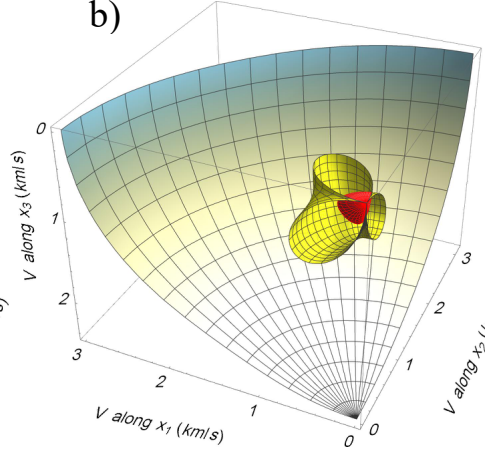

c)

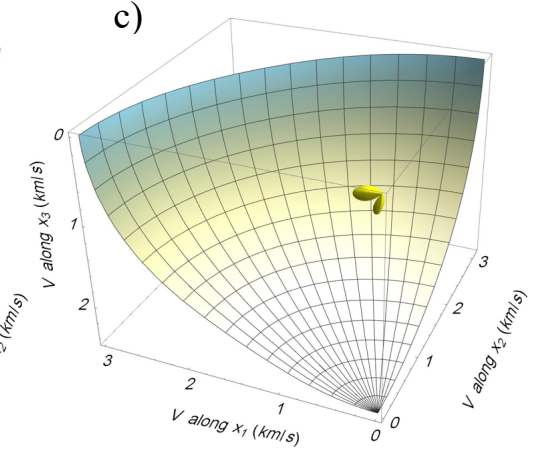

e)

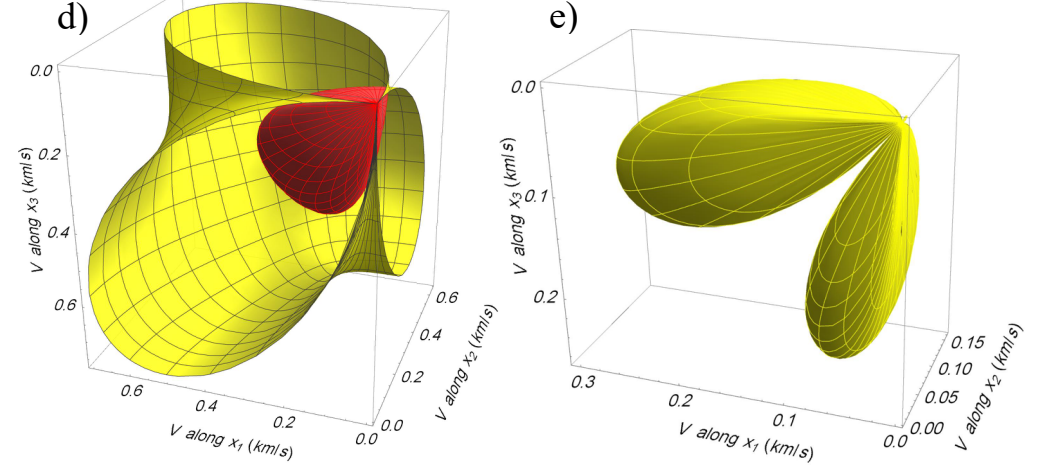

Figure 2. Phase velocity surfaces depicted for the Standard ORT model of Schoenberg and Helbig (1997; Table 1). a) Elastic case, b) conventional acoustic assumption of Alkhalifah (2003), c) the new acoustic assumption, d) enlarged version of the remaining S-waves after the conventional acoustic, and e) enlarged version of the remaining S-wave after the new acoustic assumption (note that in other models may no S-wave remain in the new acoustic case). The P, S1, and S2 waves are shown in grayscale, red, and yellow, respectively.
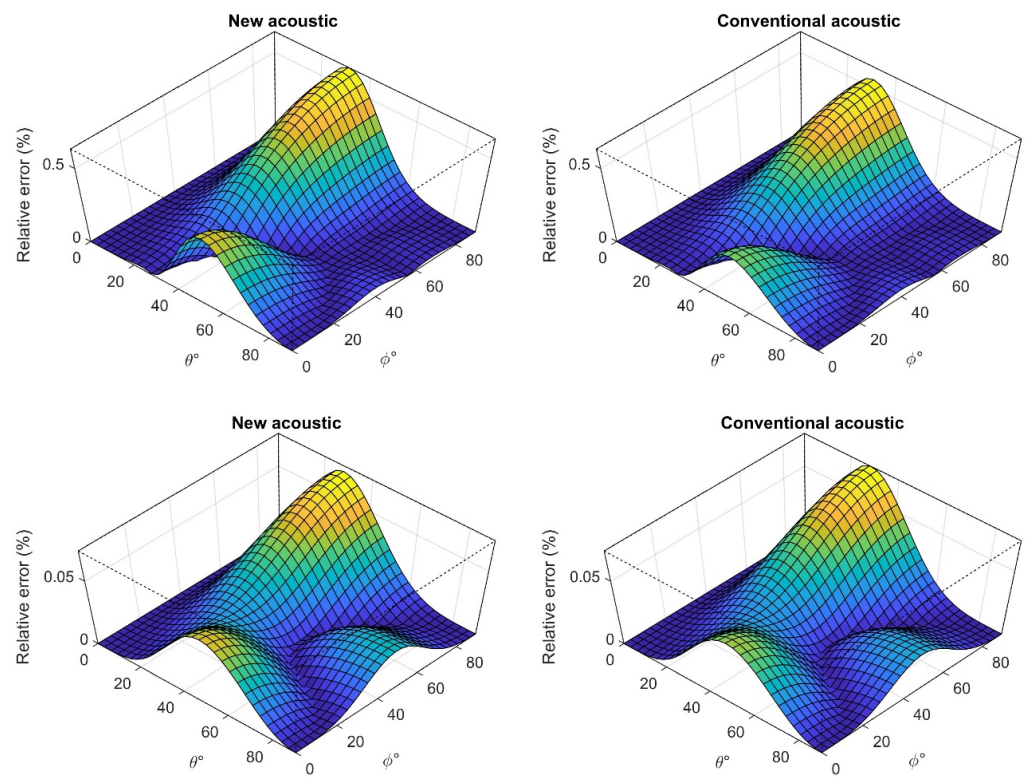

Figure 3. The absolute relative error of exact phase velocity under the new and conventional acoustic assumptions, when compared to elastic case. The upper row shows a test on the Standard ORT model (in Figure 2), the lower row shows the same for the Model 1 of Mah and Schmitt (2003; Table 1). The 
maximum errors in the Standard model are 0.62, $0.55 \%$, and in the Model 1 are $0.068,0.072 \%$, for the new and conventional acoustic, respectively.
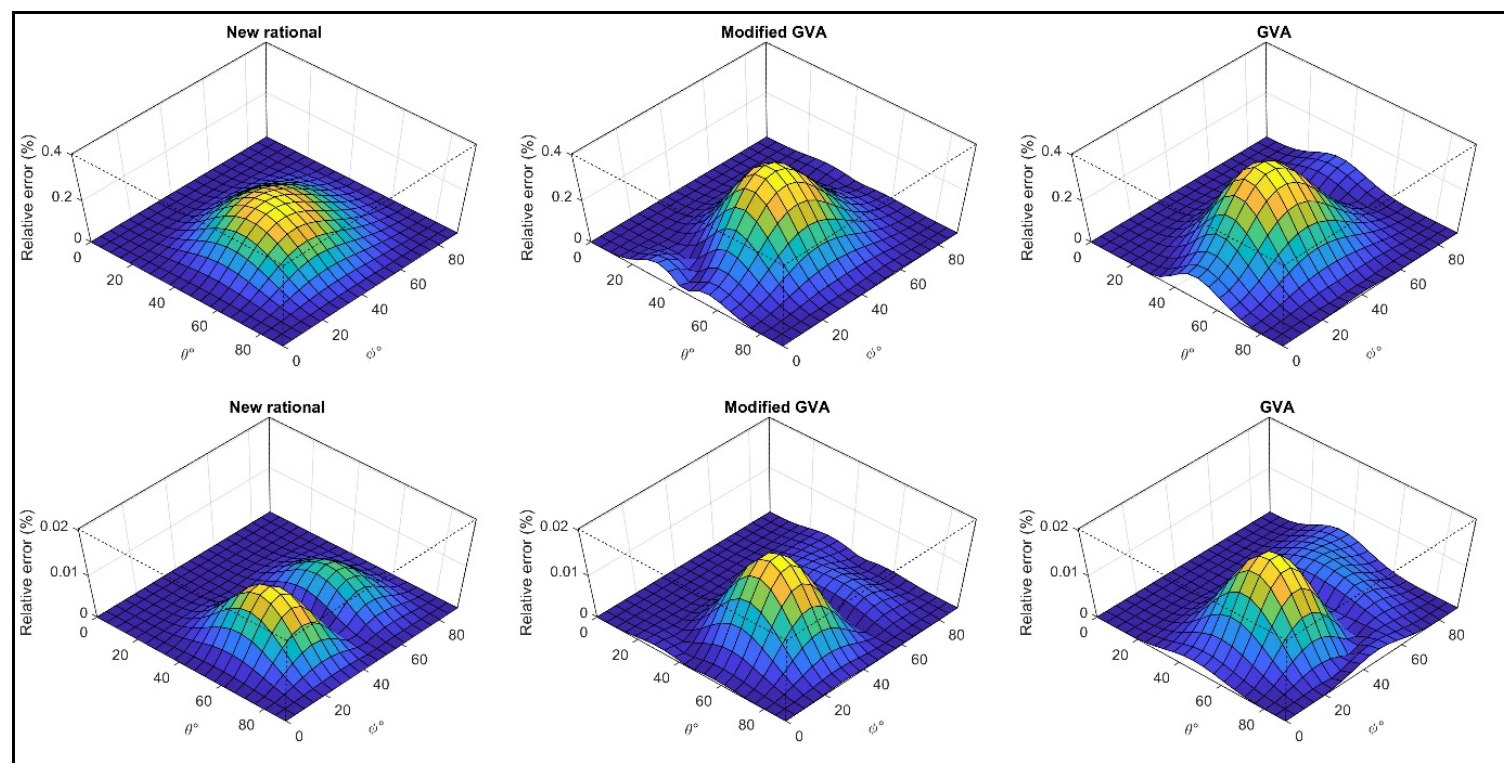

Figure 4. Absolute relative errors of the new rational phase velocity approximation (equation (3)), the modified GVA (in Appendix F), and the original GVA (Stovas and Fomel 2019), compared to exact values in the new acoustic ORT media. The upper row belongs to the Standard Model, and lower row to the Model 1 (Table 1). The maximum errors in the Standard model are 0.23, 0.39, 0.4 \%, and in the Model 1 are $0.015,0.02,0.02 \%$, for the new rational approximation, modified GVA, and GVA, respectively.
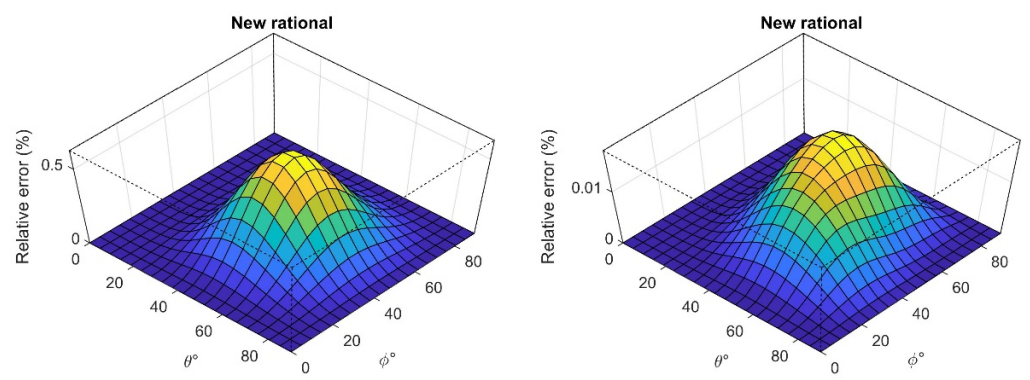

Figure 5. Absolute relative errors of the new rational slowness surface (equation (4)), the left panel is in the Standard Model, and the right panel in Model 1 (Table 1). Due to the normalization, the error surfaces in here can be compared with Figure 4 . The maximum errors are $0.6 \%$ and $0.015 \%$ from left to right. 
a)

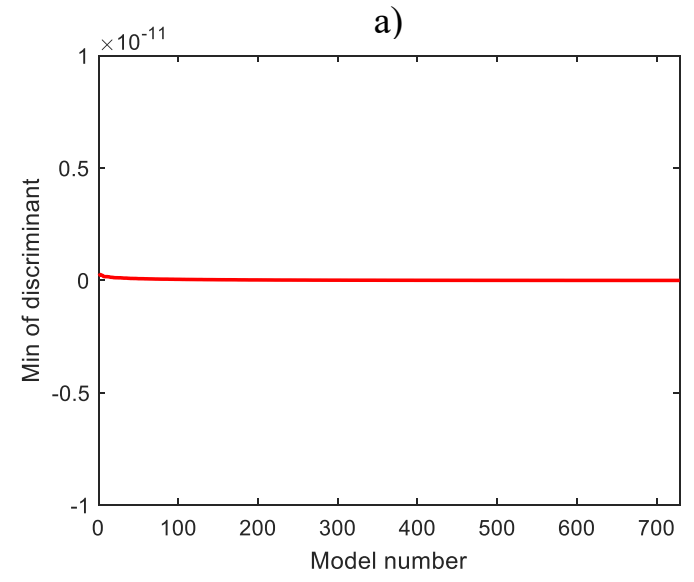

c)

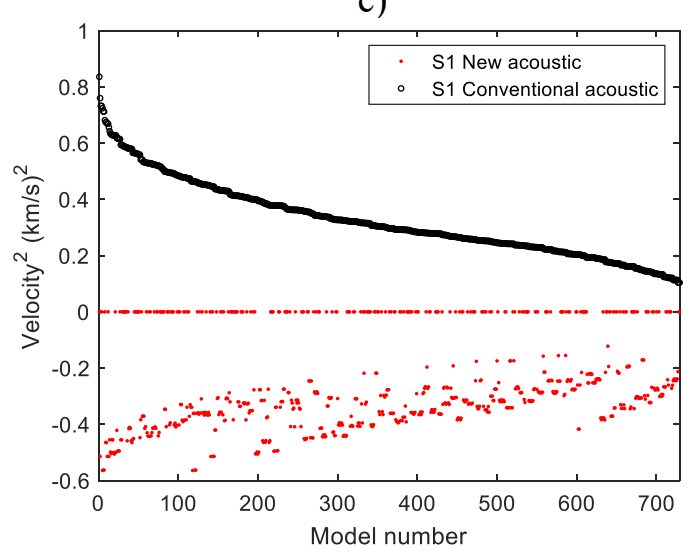

b)

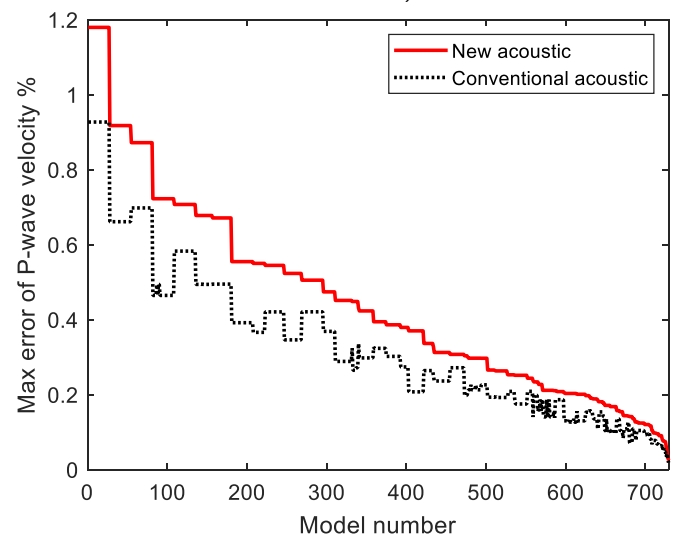

d)

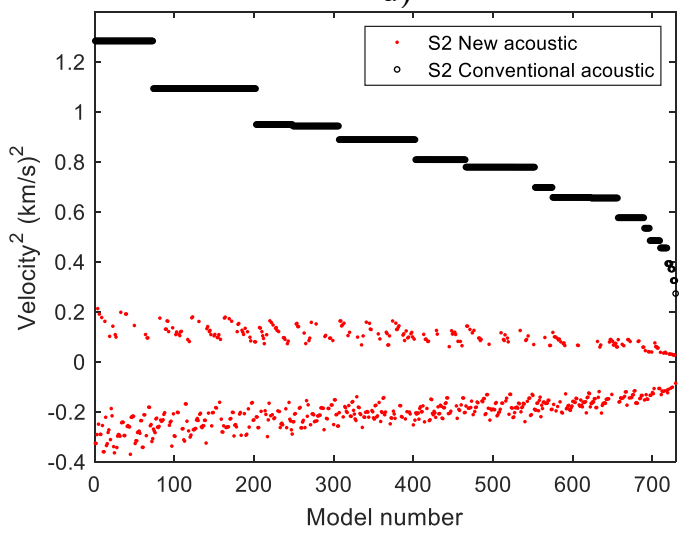

Figure 6. Numerical analysis of the new acoustic assumption, using multiple models. a) Minimum of discriminant, which is nonnegative in all models. b) The maximum of the absolute relative difference between the P-wave phase velocities in acoustic and elastic media. c) The maximum value of phase velocity squared for the $\mathrm{S} 1$ artefact; in the new acoustic assumption, it is stopped in all models. d) The maximum value of phase velocity squared for the S2 artefact; negative values show it is completely stopped, positive values show its maximum value is slowed compared to the conventional acoustic assumption. Models are differently sorted in each part.

a)

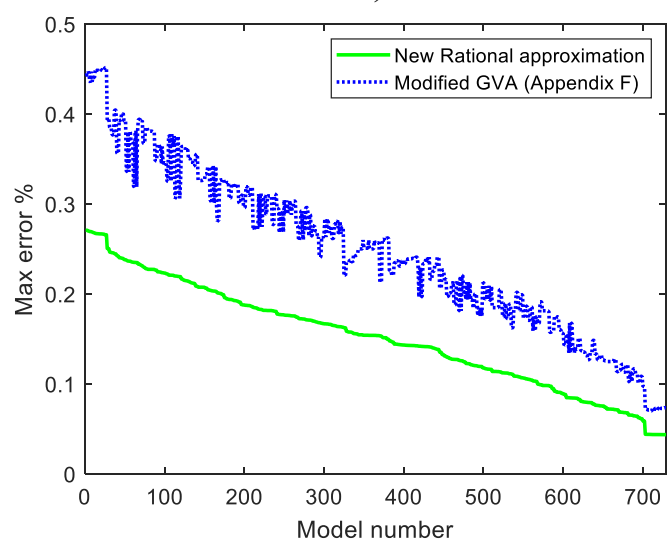

b)

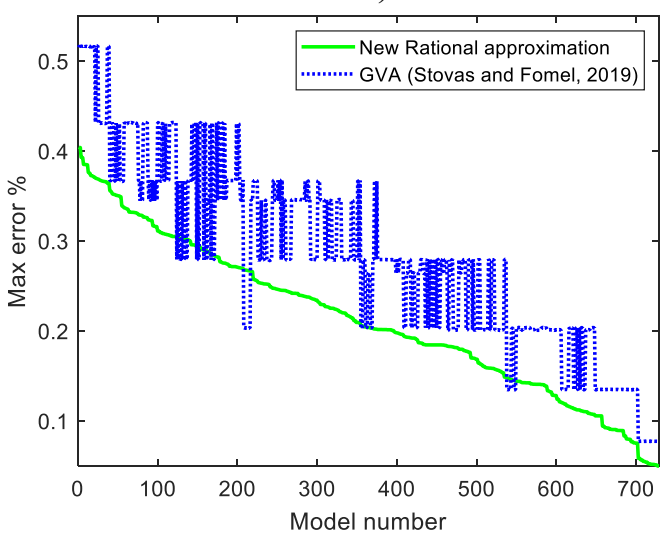

Figure 7. Accuracy of the new rational phase velocity approximation (equation (3)), compared to the exact values in the new acoustic assumption (a), and in the conventional acoustic assumption (b). The modified GVA in (a) is presented in Appendix F. 
a)

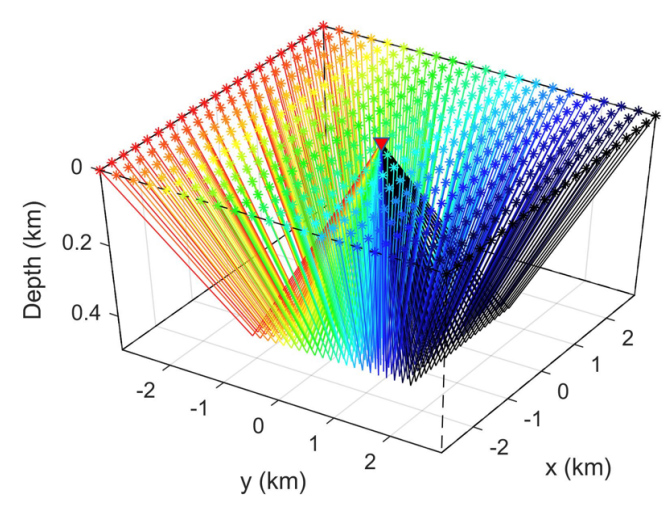

b)

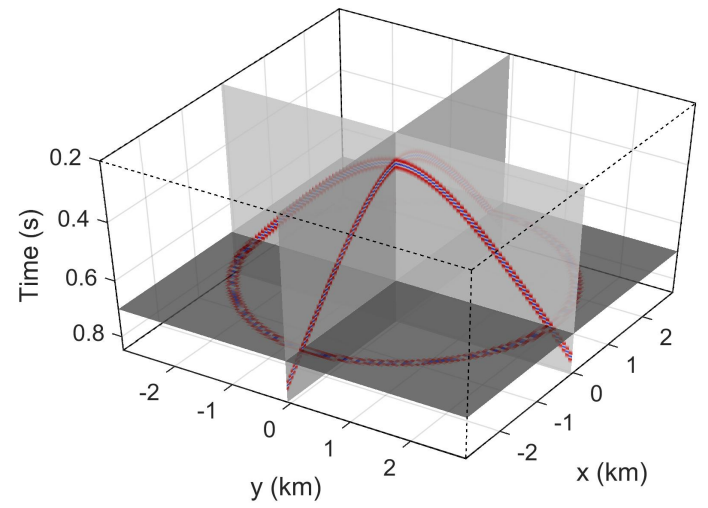

c)

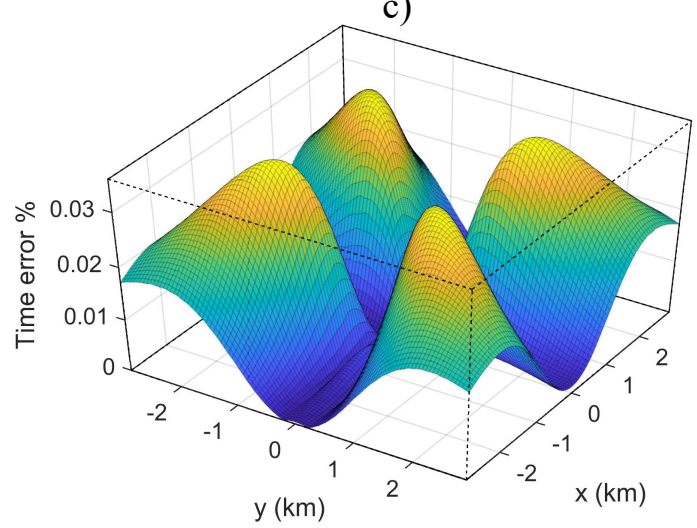

Figure 8. Simple ray tracing in the ORT Model 1 with positive $\eta_{1}$ (Table 1), using the proposed rational equation (4). a) Representative rays, b) modelled cube, c) relative traveltime difference between the modelled data by the proposed rational and the conventional acoustic equations. The maximum offset to depth ratio is 8.2 . 